\title{
Combined Treatment of Alendronate and Low-Intensity Pulsed Ultrasound (LIPUS) Increases Bone Mineral Density at the Cancellous Bone Osteotomy Site in Aged Rats: A Preliminary Study.
}

Aonuma H, Miyakoshi N, Kasukawa Y, Kamo K, Sasaki H, Tsuchie H, Segawa T, Shimada Y

Department of Orthopedic Surgery, Akita University Graduate School of Medicine, 1-1-1 Hondo, Akita 010-8543, Japan

\section{ABSTRACT}

Introduction: During fracture healing, alendronate encourages callus volume by inhibiting bone resorption, whereas low-intensity pulsed ultrasound (LIPUS) enhances bone regeneration by promoting an anabolic response.

Methods: In the present study, 9-month-old Sprague-Dawley rats, with a unilateral proximal tibial osteotomy, were treated with alendronate (daily, $1 \mu \mathrm{g} / \mathrm{kg}$ ) plus sham-LIPUS $(\mathrm{n}=14$ ), saline plus LIPUS (20 $\mathrm{min} / \mathrm{day})(\mathrm{n}=18$ ), alendronate plus LIPUS ( $\mathrm{n}=16$ ), or saline plus shamLIPUS as a control $(n=13)$ for 4 weeks. The rats were then examined for changes in bone mineral density (BMD) during metaphyseal bone repair.

Results: The combined therapy significantly increased BMD at the osteotomy site at 4 weeks ( $p<0.001$ ) compared with the control, without affecting the contralateral, non-osteotomized tibia. Both alendronate and LIPUS alone also exerted a positive, albeit less, effect on BMD in the affected limb ( $p<0.001$ and $p=0.006$, respectively).

Conclusion: Alendronate and LIPUS cooperate to enhance BMD during metaphyseal bone healing.

Keywords: LIPUS, bisphosphonate, Bone mineral density

\section{INTRODUCTION}

Osteoporosis, a progressive systemic skeletal disease, is characterized by low bone mass and micro-architectural disturbances in the bone tissue. This disease poses a major concern for the public health system, with disability and death stemming from the increased risk of fragility fractures in osteoporotic patients. ${ }^{1,2}$ Unfortunately, despite advances in preventative fracture treatment, the incidence of osteoporotic fractures seems to be rising along with an aging population. ${ }^{3}$ Therefore, there is a growing need to discern better ways to not only prevent, but also treat, fractures linked to osteoporosis. One of the most important factors to consider when performing surgery for the treatment of an osteoporotic fracture is the local condition of the bone at the fracture site, as reflected by the bone mineral density (BMD). Bisphosphonates, the most clinically important class

\footnotetext{
Correspondence:

Dr. Yuji Kasukawa,

Department of Orthopedic Surgery

Akita University Graduate School of Medicine

1-1-1 Hondo, Akita 010-8543, Japan

Tel: $+81-18-884-6148$

Fax: +81-18-836-2617

E-mail:kasukawa@doc.med.akita-u.ac.jp
} 
of anti-resorptive agents, prevent subsequent fracture after a fragility fracture, as well as reduce the risk of an initial fracture by increasing or maintaining the BMD in osteoporotic patients; these effects were later attributed to the potent inhibitory effect of bisphosphonates on bone resorption. ${ }^{4,5,6}$ However, the effect of bisphosphonates on the BMD at metaphyseal fracture sites is still unknown. On the other hand, low-intensity pulsed ultrasound (LIPUS), which is widely used in the clinical setting, has been shown to accelerate fracture healing by promoting an anabolic response. ${ }^{7}$ It has been reported that LIPUS works to increase callus mineral density along with callus maturation at the diaphyseal fracture site. ${ }^{8}$ However, it is still unclear whether LIPUS increases the BMD at both the fractured and unfractured metaphyseal sites. Thus, it is hypothesized that treatment with bisphosphonate and LIPUS might have an additive effect during metaphyseal fracture repair, and their concomitant use may offer an alternative treatment for osteoporotic metaphyseal fractures. The purpose of this study was to investigate the effects of the bisphosphonate, alendronate, in conjunction with LIPUS on the BMD during metaphyseal repair using a rat proximal tibial osteotomy model.

\section{METHODS}

\section{Animal preparation}

Nine-month-old female Sprague-Dawley rats (Charles River Laboratory Inc., Kanagawa, Japan) were housed in a controlled environment at $22^{\circ} \mathrm{C}$ with a 12-h light/ dark cycle. The rats were permitted free access to water and standard feed (CE-2, Clea Japan Inc., Tokyo, Japan). All rats underwent mid-sagittal osteotomy on the right proximal tibia. Briefly, a lateral parapatellar incision was created in the knee joint through the proximal half of the tibia. Using a powered saw (Yoshida Medical Inc., Japan), an osteotomy was then created in the upper one-fourth of the proximal tibia, without extending to the caudal cortex, as previously reported. ${ }^{9}$ The osteotomized tibiae were closed using a non-absorbable suture. After surgery, the rats were allowed to move freely; those rats which displayed an abnormal gait or impaired locomotion were excluded from later assessment.

\section{Experimental design}

Rats were randomly assigned into one of four groups: alendronate group (alendronate plus sham-LIPUS; $\mathrm{n}=$ 14), LIPUS group (saline vehicle plus LIPUS; $n=18$ ), combined group (alendronate plus LIPUS; $\mathrm{n}=16$ ) and the control group (saline vehicle plus sham-LIPUS; $\mathrm{n}=$ 13). A saline vehicle was used in place of alendronate in the LIPUS and the control groups. Daily drug/vehicle administration and LIPUS/sham-LIPUS interventions on both osteotomized and non-osteotomized tibiae were initiated on the third day after the osteotomy and continued until sacrifice. Body weights were measured prior to the osteotomy and once per week after the osteotomy to adjust the dosage of alendronate. For LIPUS or sham-LIPUS exposure each day, rats were given general anesthesia with intra-peritoneal ketamine (20 mg/kg) (Sankyo, Tokyo, Japan) and xylazine (1.5 mg/kg) (ZENOAQ, Fukushima, Japan). For the administration of sham-LIPUS, rats were subjected to the same procedure as LIPUS, without the ultrasound activated. Rats were sacrificed under anesthesia four weeks after the osteotomy, and their bilateral tibiae were preserved in $10 \%$ neutral buffered formalin for evaluation. All animal experiments were approved by the "Guidelines for Animal Experiment" of Akita University School of Medicine.

\section{Alendronate administration}

Rats in the alendronate and the combined groups received $1 \mu \mathrm{g} / \mathrm{kg}$ alendronate (Teiroc Injection $10 \mathrm{mg}$, Teijin Pharma, Tokyo, Japan) prepared in saline at a concentration of $0.02 \mathrm{mg} / \mathrm{ml}$ subcutaneously. Rats in the LIPUS and the control groups received a subcutaneous $0.2 \mathrm{ml}$ saline injection as a vehicle control.

\section{Ultrasound intervention}

LIPUS was provided by a Sonic Accelerated Fracture Healing System (SAFHS 2000J; Teijin Pharma, Tokyo, Japan). The ultrasound signals were transferred for 20 min per day with a spatial average-temporal average (SATA) intensity of $30 \mathrm{~mW} / \mathrm{cm} 2$; these are the clinically utilized settings recommended for this device. The transducer that generates the signals was applied to the antero-medial side of osteotomized and non-osteotomized tibiae. Rats receiving sham-LIPUS underwent the same procedure as rats receiving LIPUS, without the ultrasound activated.

\section{Measurement of BMD}

The bone mineral density (BMD) of both excised tibiae was measured by dual-energy X-ray absorptiometry (Hologic QDR-4500, Hologic, MA, USA) in the anteroposterior view. The bones were scanned in the "small animal" scan mode, with the "regional highresolution" scan option. The region of interest (ROI) was taken $20 \mathrm{~mm}$ from the tip of the proximal tibia and spanned across the total width of the bone(Figure 1). 


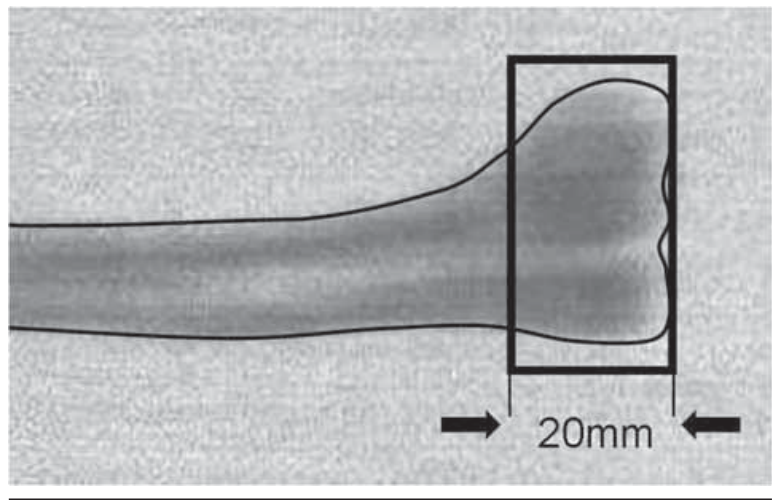

Figure 1. The region of interest (ROI) measured 20 $\mathrm{mm}$ from the proximal tip of tibia and included the entire surface area within that region.

\section{Statistical analysis}

Body weight and BMD are represented as mean \pm standard deviation (SD). Statistical differences among treatment groups were compared using Scheffe's posthoc test for multiple comparisons in analysis of variance (ANOVA). The body weight change from pre-osteotomy to four weeks post-osteotomy was evaluated with a paired t-test. Two-factor factorial ANOVA was used to evaluate the effect of alendronate and LIPUS alone or in combination on BMD. Statistical analyses were performed using Stat View 5.0J for Windows (SAS Institute, NC, USA).

\section{RESULTS}

\section{Body weight changes}

Table 1. shows body weight changes in rats. There was a significant decrease in the body weight of all of the rats during the experimental period ( $p<0.001$ ). However, the body weights of the rats before the osteotomy were the same across all the experimental groups, as were the body weights of the rats four weeks after the osteotomy.

Table1. Body weight changes in rats

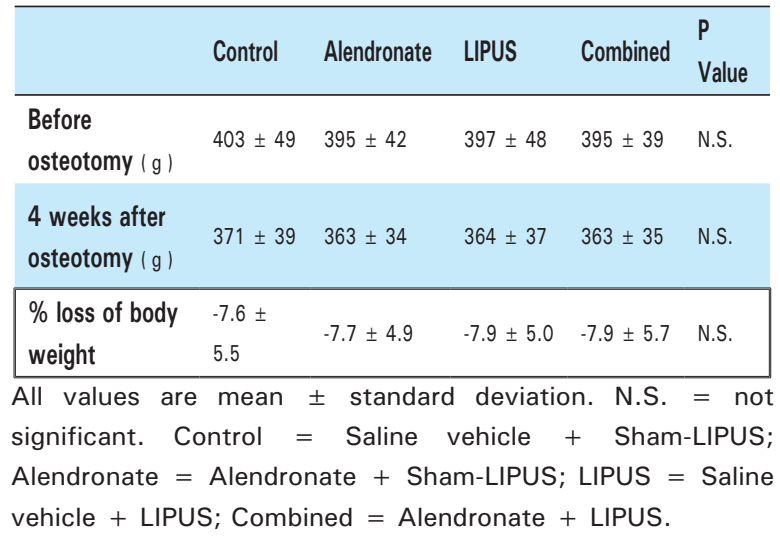

\section{Bone Mineral Density}

The combined treatment of alendronate and LIPUS significantly increased BMD at the osteotomy site by $12 \%$, as compared with the control group $(p<0.001)$. In addition, the two-factor factorial ANOVA showed that there was a significant effect of alendronate and LIPUS on BMD when administered separately $(p<$ 0.001 and $p=0.006$, respectively). In contrast, no significant differences were seen in contralateral nonosteotomized tibiae across the various experimental groups in the multiple comparison, as compared with rats in the control group (Figure 2).

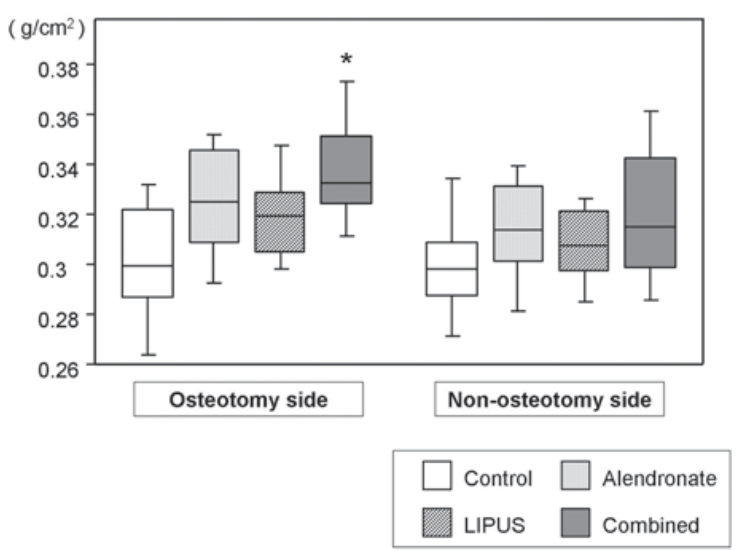

Figure 2: Control = Saline vehicle + Sham-LIPUS; Alendronate $=$ Alendronate + sham-LIPUS; LIPUS $=$ Saline vehicle + LIPUS; Combined $=$ Alendronate + LIPUS. ${ }^{*} p<0.001$ calculated in multiple comparison using Scheffe's post-hoc test.

\section{DISCUSSION}

During osteoporotic metaphyseal bone repair, the choice of an implant is affected by the degree of bone stock in the patient and the quality of the trabecular network. ${ }^{10,11}$ Consequently, BMD is an important factor in achieving adequate implant fixation during surgery. ${ }^{12,13}$ In this study, BMD was significantly higher in rats treated with the combination of alendronate and LIPUS as compared with that in the control group, suggesting that these treatment strategies are able to improve the local condition of the cancellous bone. Furthermore, our results suggest that the positive effects on BMD by these two interventions are independent of each other, implying an additive effect when administered together. Based on these results, the combined treatment of alendronate and LIPUS is an ideal choice for promoting metaphyseal fracture healing and improving screw fixation. Additionally, the increase in BMD was observed 
only at the osteotomy side, suggesting that alendronate and LIPUS have, at least in part, fracture-site specificity in their action.

It is well-known that bisphosphonates increase the BMD of post-menopausal osteoporotic women. Several fracture models using ovariectomized rats have shown that bisphosphonate increases BMD at the diaphysis of a long bone. ${ }^{14,15,16}$ In the present study, alendronate increased BMD in the aged rats after metaphyseal osteotomy of the tibia. Matos et al. showed that one of the most potent bisphosphonates, zoledronate, retained the amount of trabecular bone during repair by stimulating primary bone production and inhibiting bone remodeling. ${ }^{17}$ Here, too, it is possible that alendronate increased BMD by preserving the amount of pre-existing bone at the osteotomy site.

LIPUS is often used to stimulate fracture healing after osteosynthesis surgery, particularly where there is delayed union or non-union. Other studies suggest that LIPUS has a beneficial effect on bone healing by positively promoting signal transduction, gene expression, blood flow, and tissue modeling and remodeling. ${ }^{18}$ In fracture studies, LIPUS treatment appears to shorten the healing period and increase callus mineral density. ${ }^{8,19}$ Based on these reports, we hypothesize that LIPUS exposure increased BMD by accelerating regenerated bone maturation at the osteotomy site.

Body weight is another contributor to BMD, as weight is positively associated with mechanical stress. In the present study, the body weight of all rats was decreased during the four weeks treatment, irrespective of the treatment group allocated. It is unlikely that the treatment affected the body weight, as the magnitudes of body weight loss were similar among the four groups, including the control that did not receive alendronate or LIPUS. Therefore, the loss of body weight was likely caused by the daily general anesthesia during the experimental periods, rather than the treatment modalities. In a clinical setting, general anesthesia is not required for treatment with alendronate and LIPUS, and therefore the influence of body weight loss should be negligible in a clinical setting.

There were several limitations in this preliminary study. One of these limitations was that we only evaluated $\mathrm{BMD}$ at the proximal tibia during cancellous bone repair. Further studies are required to measure the rate of bone union, using histopathological evaluation, and bone strength via mechanical testing at the cancellous bone osteotomy site after treatment. These tests are important to more accurately evaluate the effects of combined treatment of alendronate and LIPUS on cancellous bone repair.

\section{CONCLUSIONS}

In conclusion, this preliminary study showed that the combination of alendronate and LIPUS increased BMD at the osteotomy site, in an independent manner. Alendronate may act by increasing bone volume around the osteotomy site, whereas LIPUS facilitates maturation of the regenerated bone. Their combined use appears to have an additive effect on the BMD during cancellous bone healing, although further studies are warranted to confirm these effects.

\section{ACKNOWLEDGMENTS}

We thank Teijin Pharma, Tokyo, Japan for kindly supplying the alendronate and SAFHS. We also thank Ms. K. Sakamoto and R. Kamoya for their technical assistance.

\section{REFERENCES}

1. Johnell O, Kanis JA, Odén A, Sernbo I, Redlund-Johnell I, Petterson C, et al. Mortality after osteoporotic fractures. Osteoporos Int. 2004, 15:38-42.

2. Salaffi F, Cimmino MA, Malavolta N, Carotti M, Di Matteo $\mathrm{L}$, Scendoni $\mathrm{P}$, et al. The burden of prevalent fractures on health-related quality of life in postmenopausal women with osteoporosis: the IMOF study. J Rheumatol. 2007, 34:1551-60.

3. Holt G, Smith R, Duncan K, Hutchison JD, Reid D. Changes in population demographics and the future incidence of hip fracture. Injury. 2009, 40:722-26.

4. van der Poest Clement E, van Engeland M, Adèr H, Roos JC, Patka P, Lips P. Alendronate in the prevention of bone loss after a fracture of the lower leg. J Bone Miner Res. 2002, 17:2247-55.

5. Hochberg MC, Thompson DE, Black DM, Quandt SA, Cauley J, Geusens P, et al. Effect of alendronate on the agespecific incidence of symptomatic osteoporotic fractures. J Bone Miner Res. 2005, 20:971-76.

6. Eriksen EF, Lyles KW, Colón-Emeric CS, Pieper CF, Magaziner JS, Adachi JD, et al. Antifracture efficacy and reduction of mortality in relation to timing of the first dose of zoledronic acid after hip fracture. J Bone Miner Res. 2009, 24:1308-13.

7. Claes L, Willie B. The enhancement of bone regeneration by ultrasound. Prog Biophys Mol Biol. 2007, 93:384-98.

8. Hantes ME, Mavrodontidis AN, Zalavras CG, Karantanas AH, Karachalios T, Malizos KN. Low-intensity transosseous ultrasound accelerates osteotomy healing in a sheep fracture model. J Bone Joint Surg Am. 2004, 86-A: 2275-82. 
9. Nozaka K, Miyakoshi N, Kasukawa Y, Maekawa S, Noguchi $\mathrm{H}$, Shimada Y. Intermittent administration of human parathyroid hormone enhances bone formation and union at the site of cancellous bone osteotomy in normal and ovariectomized rats. Bone. 2008, 42:90-97.

10. Kim WY, Han CH, Park JI, Kim JY. Failure of intertrochanteric fracture fixation with a dynamic hip screw in relation to preoperative fracture stability and osteoporosis. Int Orthop. 2001, 25:360-62.

11. Giannoudis P, Tzioupis C, Almalki T, Buckley R. Fracture healing in osteoporotic fractures: is it really different? A basic science perspective. Injury. 2007, 38 Suppl 1:S90-99.

12. Okuyama K, Abe E, Suzuki T, Tamura Y, Chiba M, Sato K. Influence of bone mineral density on pedicle screw fixation: a study of pedicle screw fixation augmenting posterior lumbar interbody fusion in elderly patients. Spine J. 2001, 1:402-407.

13. Seebeck J, Goldhahn J, Morlock MM, Schneider E. Mechanical behavior of screws in normal and osteoporotic bone. Osteoporos Int. 2005, 16 Suppl 2:S107-11.

14. Li J, Mori S, Kaji Y, Mashiba T, Kawanishi J, Norimatsu H. Effect of bisphosphonate (incadronate) on fracture healing of long bones in rats. J Bone Miner Res. 1999, 14:969-79.
15. Amanat N, McDonald M, Godfrey C, Bilston L, Little D. Optimal timing of a single dose of zoledronic acid to increase strength in rat fracture repair. J Bone Miner Res. 2007, 22:86776.

16. Cao Y, Mori S, Mashiba T, Kaji Y, Manabe T, Iwata K, et al. 1Alpha,25-dihydroxy-2beta(3-hydroxypropoxy)vitamin D3 (ED-71) suppressed callus remodeling but did not interfere with fracture healing in rat femora. Bone. 2007, 40:132-39.

17. Matos MA, Tannuri U, Guarniero R. The effect of zoledronate during bone healing. J Orthop Traumatol. 2010, 11:7-12.

18. Khan Y, Laurencin CT. Fracture repair with ultrasound: clinical and cell-based evaluation. J Bone Joint Surg Am. 2008, 90 Suppl 1:138-44.

19. Shakouri K, Eftekharsadat B, Oskuie MR, Soleimanpour J, Tarzamni MK, Salekzamani Y, et al. Effect of low-intensity pulsed ultrasound on fracture callus mineral density and flexural strength in rabbit tibial fresh fracture. J Orthop Sci. 2010, 15:240-44 